\title{
SST spatial anisotropic covariances from METOP-AVHRR data
}

\author{
Pierre Tandeo ${ }^{\mathrm{a}, *}, 1$, Emmanuelle Autret $^{\mathrm{b}, 1},{\text { Bertrand } \text { Chapron }^{\mathrm{b}} \text {, Ronan Fablet }{ }^{\mathrm{a}} \text {, René Garello }}^{\mathrm{a}}$
}

\author{
a Institut Mines-Telecom, Telecom Bretagne, CNRS UMR 6285 LabSTICC, Pôle CID, Technopôle Brest Iroise, \\ CS 83818, 29238 BREST Cedex, France \\ ${ }^{b}$ IFREMER, Laboratoire d'océanographie spatiale, 29280 Plouzane, France \\ ${ }^{1}$ Both authors equally contributed to this work.
}

*: Corresponding author : Pierre Tandeo, tel.: + 33229001570 ;

email address : pierre.tandeo@telecom-bretagne.eu

\begin{abstract}
:
The Advanced Very High Resolution Radiometer (AVHRR) instrument on-board the METOP satellite is designed to provide very accurate measurements of Sea Surface Temperature (SST). In this work, using one year of METOP-AVHRR data and a geostatistical approach, we characterize the spatial anisotropy and non-stationarity of the SST variability using oriented ellipsoids. The method is also able to separate the true SST variability from the artificial error introduced by the METOP-AVHRR sensor. These spatial parameters are then used for producing variability atlases (available on-line) over the whole ocean.
\end{abstract}

\section{Highlights}

Completely observation-based approach - Anisotropy of the SST spatial variability is fitted by oriented ellipsoids. Regions with high isotropic or anisotropic behavior are highlighted. Regions with high measurement errors of the METOP-AVHRR are highlighted. The spatial variability parameters for the global Ocean are available online.

Keywords: SST ; METOP-AVHRR ; Spatial variability ; Anisotropy

\section{Introduction}

Sea Surface Temperature (SST) is a key geophysical variable for many applications (see e.g. Donlon et al., 2002 and reference therein). For instance, global-scale, complete and realistic SST fields are key input data in the Ocean General Circulation Models (OGCMs). One may also cite the use of SST data to track, characterize and reconstruct mesoscale dynamics (cf. Isern-Fontanet et al., 2006, Klein et al., 2009 and Tandeo et al., 2013). In this respect, satellite sensing data provide invaluable globalscale data with relatively high spatial 
and temporal resolutions. Such data are however vulnerable to various atmospheric contaminations (e.g., cloud cover, aerosols, heavy rains, etc...) and they may present large missing data rates. Therefore, spatial and temporal interpolation techniques are necessary to reconstruct the hidden information (see e.g. Reynolds et al. (2007)). Understanding and modeling of mesoscale SST variability appears crucial to provide interpolation priors.

The temporal variability of the SST has been investigated for short time scales from in situ data (cf. Wainer et al. (2003)) and from remote sensing sources (cf. Tandeo et al. (2011)) but, its spatial variability, especially spatial anisotropy and non-stationarity, remains poorly explored. Whereas regional studies addressed the isotropic spatial variability of the SST, (e.g. Gohin and Langlois (1993) in the bay of Biscay), evidences for local spatial anisotropies have been reported for SST fields in some studies (e.g. Park and Chung (1999) in the Sea of Japan) as well as for other geophysical variables such as ocean colour (cf. Doney et al. (2003)). However, to our knowledge, no study has explored thoroughly at a global-scale these mesoscale spatial SST variabilities. This study relies on geostatistic tools to address these issues from the analysis of the Advanced Very High Resolution Radiometer (AVHRR) instrument on-board the METOP-A satellite (cf. Le Borgne et al. (2007); O\&SI SAF (2013)). Using compact parametric model of the spatial covariance of the SST fields and the associated model fitting scheme, we produce observation-based atlases of the mesoscale spatial variability of the SST for the global Ocean, in terms of local anisotropy, spatial scales and residual variances. These atlases provide new insights on global and regional mesoscale ocean dynamics and new priors for missing data interpolation.

This paper is organized as follows. The METOP-AVHRR SST data and the geostatistical methodology are introduced in Section 2. We discuss the results obtained in the Malvinas current and the global Ocean in Section 3. Section 4 further discusses our key contributions and prospects for future work.

\section{Method}

\subsection{METOP-AVHRR data}

METOP-A satellite has a high resolution coverage of the world Ocean. The data are delivered in satellite projection at full resolution and remapped onto a regular $0.05^{\circ} \times 0.05^{\circ}$ global grid every 12 hours (see O\&SI SAF (2013) for more details). METOP-AVHRR is one of the most efficient SST data source due to the high quality measurements and the number of observations (cf. Le Borgne et al. (2007)). In this study, we only use night-time data to avoid the vari- 
ability due to the diurnal cycle, good-quality pixel data corresponding to best proximity confidence levels (flags 4 and 5). We considered the year 2008 and the global Ocean. The resulting METOP SST fields are clearly non-stationary with important seasonal components. The non-stationary components have complex features and we could not find any appropriate parametric model to describe them. To remove these components, we used the daily $0.25^{\circ}$ resolution Optimal Interpolation Version 2 (OIV2) analysis provided by the National Climatic Data Center (NCDC). This analysis is derived from different satellite sources independent of METOP data (cf. Reynolds et al. (2007)). We assume that they provide a good estimate of the low-variations of the SST conditions. In this paper, our work is focused on the SST anomaly given by the difference between the METOP and the corresponding OIV2 fields. This anomaly characterizes the mesoscale (horizontal scales of $50 \mathrm{~km}$ to few hundred kilometers) non-stationary spatial variability of the SST. An example of SST anomaly field in the Malvinas current is given in Fig. 1. The white pixels correspond to flagged data due to the proximity to clouds. The use of the OIV2 fields instead of a spatial filter version of the METOP-AVHRR SSTs is motivated by the following reasons. Firstly, due to the important cloud coverage in certain regions, it is often impossible to generate realistic spatially smoothed SST fields from METOP-AVHRR. Secondly, to be consistent with a previous analysis of the temporal variability of the SST anomalies (cf. Tandeo et al. (2011)), we decided to use the same year 2008 and the same reference fields (i.e., OIV2 fields).

\subsection{Anisotropic semivariogram model}

To spatially interpolate a missing SST anomaly at a specific location $s$ noted as $Z(s)$, we need to characterize the local variability of the neighborhood. Thus, we define the semivariogram function $\gamma$ between two locations $s_{1}$ and $s_{2}$ (separated by a distance $\Delta$ ) as

$$
\begin{aligned}
2 \gamma(\Delta)= & \operatorname{Var}\left(Z\left(s_{1}\right)-Z\left(s_{2}\right)\right) \\
= & \operatorname{Var}\left(Z\left(s_{1}\right)\right)+\operatorname{Var}\left(Z\left(s_{2}\right)\right) \\
& -2 \operatorname{Cov}\left(Z\left(s_{1}\right), Z\left(s_{2}\right)\right)
\end{aligned}
$$

The process $\{Z\}$ is said to be second-order stationary if the mean is the same at each location and if its spatial covariance only depends on the spatial distance $\Delta$ between $s_{1}$ and $s_{2}$ such as

$$
\operatorname{Cov}\left(Z\left(s_{1}\right), Z\left(s_{2}\right)\right)=C(\Delta)
$$

where $C$ is the spatial covariance function. According to the previous state- 


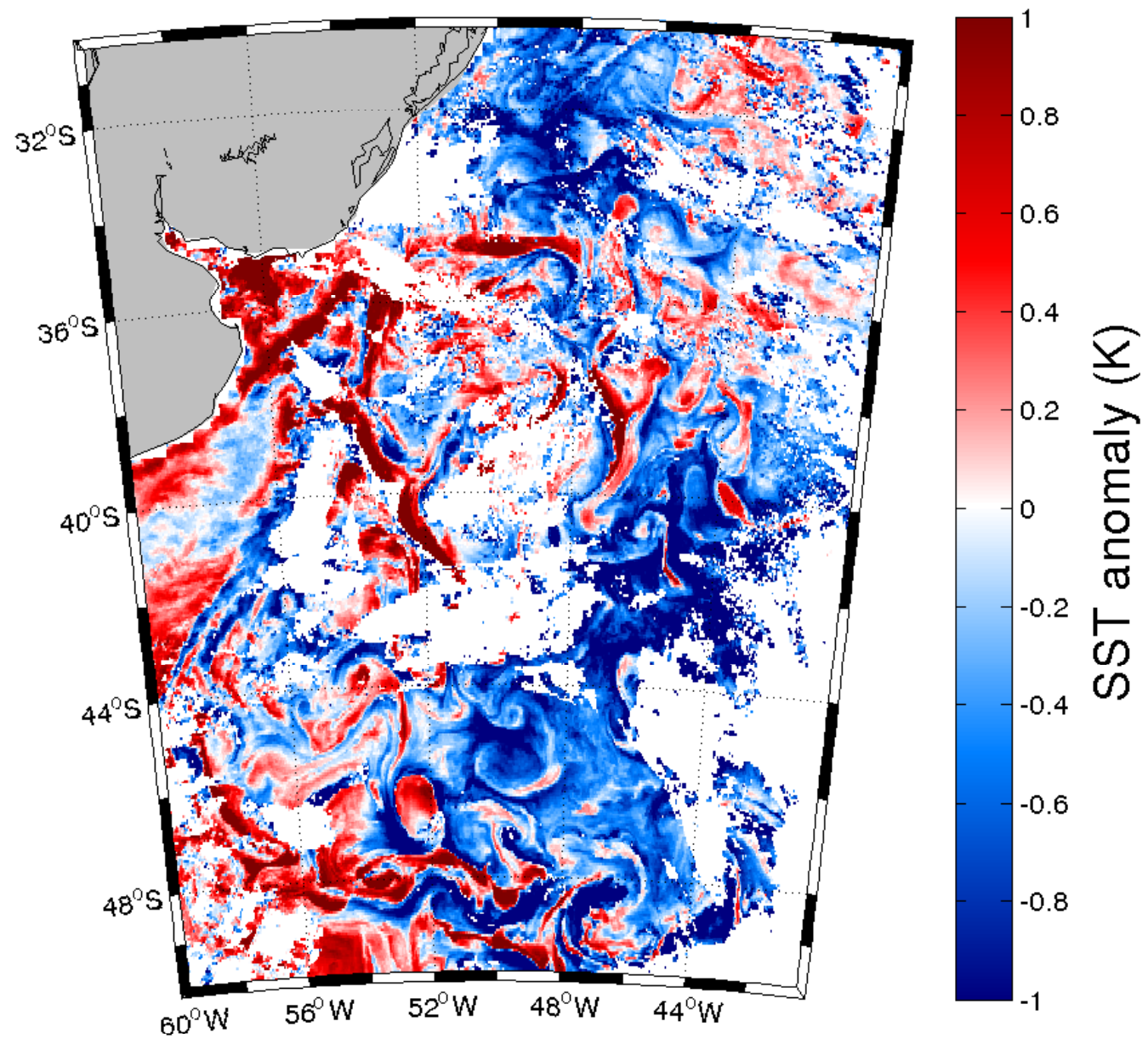

Fig. 1. Example of a SST anomaly field in the Malvinas current the $1^{\text {st }}$ of January, 2008. The anomaly is computed from the difference between the high spatial resolution METOP data and the low spatial resolution OIV2 data.

ments (1), (2) and knowing that $\operatorname{Var}\left(Z\left(s_{i}\right)\right)=C(0)$ for any spatial location $s_{i}$, we obtain

$$
\gamma(\Delta)=C(0)-C(\Delta)
$$

To account for spatial anisotropy, i.e. the existence of principal directions of variations, the spatial lag $\Delta$ (in kilometers) is decomposed according to a zonal component $\Delta_{x}$ and a meridional $\Delta_{y}$ one such as $\Delta^{2}=\Delta_{x}^{2}+\Delta_{y}^{2}$. A classical empirical estimation of the semivariogram (cf. Cressie (1993)) is given by

$$
\widehat{\gamma}\left(\Delta_{x}, \Delta_{y}\right)=\frac{\sum_{\left(s_{i}, s_{j}\right)}\left(Z\left(s_{i}\right)-Z\left(s_{j}\right)\right)^{2}}{2 n}
$$

where $n$ denotes all pairs of points $\left(s_{i}, s_{j}\right)$, for which the longitude and latitude spatial distance equal $\Delta_{x}$ and $\Delta_{y}$.

The semivariogram measures the local spatial variabilities of the process $\{Z\}$ 
as a function of the zonal and meridional distances, $\Delta_{x}$ and $\Delta_{y}$. Then, we fit a positive-definite parametric model (e.g. exponential, Gaussian, spherical, power or Matérn models) to the empirical semivariogram $\widehat{\gamma}$. In our case, according to previous analyses (not shown in this paper) and to reduce the number of parameters, we decided to use an anisotropic exponential model given by

$$
\gamma\left(\Delta_{x}, \Delta_{y}\right)=\sigma_{0}^{2}+\sigma^{2}\left(1-\exp \left(-\frac{\Delta}{L(\theta)}\right)\right)
$$

where $(\Delta, \theta)$ refers to the polar representation of $\left(\Delta_{x}, \Delta_{y}\right)$. This model involves two variance parameters: the nugget $\sigma_{0}^{2}$ which represents low spatial variations (typically instrumental uncertainties) and the sill $\sigma^{2}$ (such as $\sigma^{2}>\sigma_{0}^{2}$ ) which accounts for the stationary variance of the process. The graphical representation of the theoretical semivariogram (5) is given by an ellipse as shown in Fig. 2. This ellipse is parameterized according to the length of the minor and major axes, respectively denoted by $L_{\min }$ and $L_{\max }$ and its orientation $\phi \in(0, \pi)$ defined as the angle of the major axis with respect to the North. Given these ellipse parameters, distance $L(\theta)$ in Eq. (5) is defined as

$$
L(\theta)=\frac{L_{\min } L_{\max }}{\sqrt{L_{\max }^{2} \cos ^{2}(\theta-\phi)+L_{\min }^{2} \sin ^{2}(\theta-\phi)}}
$$

with $L_{\min } \leq L(\theta) \leq L_{\max }$ for any angular value $\theta$. Physically, the orientation $\phi$ defines the preferred direction of variation, $L_{\min } / L_{\max }$ the strength of the anisotropy. Besides $L_{\max }$ relates to the correlation range along the preferred direction of variation.

To fit the parameters of the spatial covariance, we minimize the difference between the empirical semivariogram estimates (4) and the theoretical semivariogram (5) using the weighted least squares criterion and a Gauss-Newton algorithm. This iterative procedure is initialized as follows. The minor and major ranges $L_{\min }$ and $L_{\max }$ are chosen as the Rossby radius. Then, $\phi, \sigma_{0}^{2}$ and $\sigma^{2}$ are respectively initialized as the main orientation, the minimum and maximum values of the empirical semivariogram computed in Eq. (4).

\section{Results}

The estimated parameters $\sigma_{0}^{2}$ (nugget), $\sigma^{2}$ (sill), $\phi$ (anisotropic angle), $L_{\text {min }}$ (minimum range) and $L_{\max }$ (maximum range) are discussed in this section. They are computed for a $1^{\circ} \times 1^{\circ}$ grid over the global Ocean. Locally, we can suppose that the spatial variability is second-order stationary. The computation of the empirical semivariograms proceeds as follows. For a given $1^{\circ} \times 1^{\circ}$ area, we first compute daily empirical semivariograms: we sort all locations 


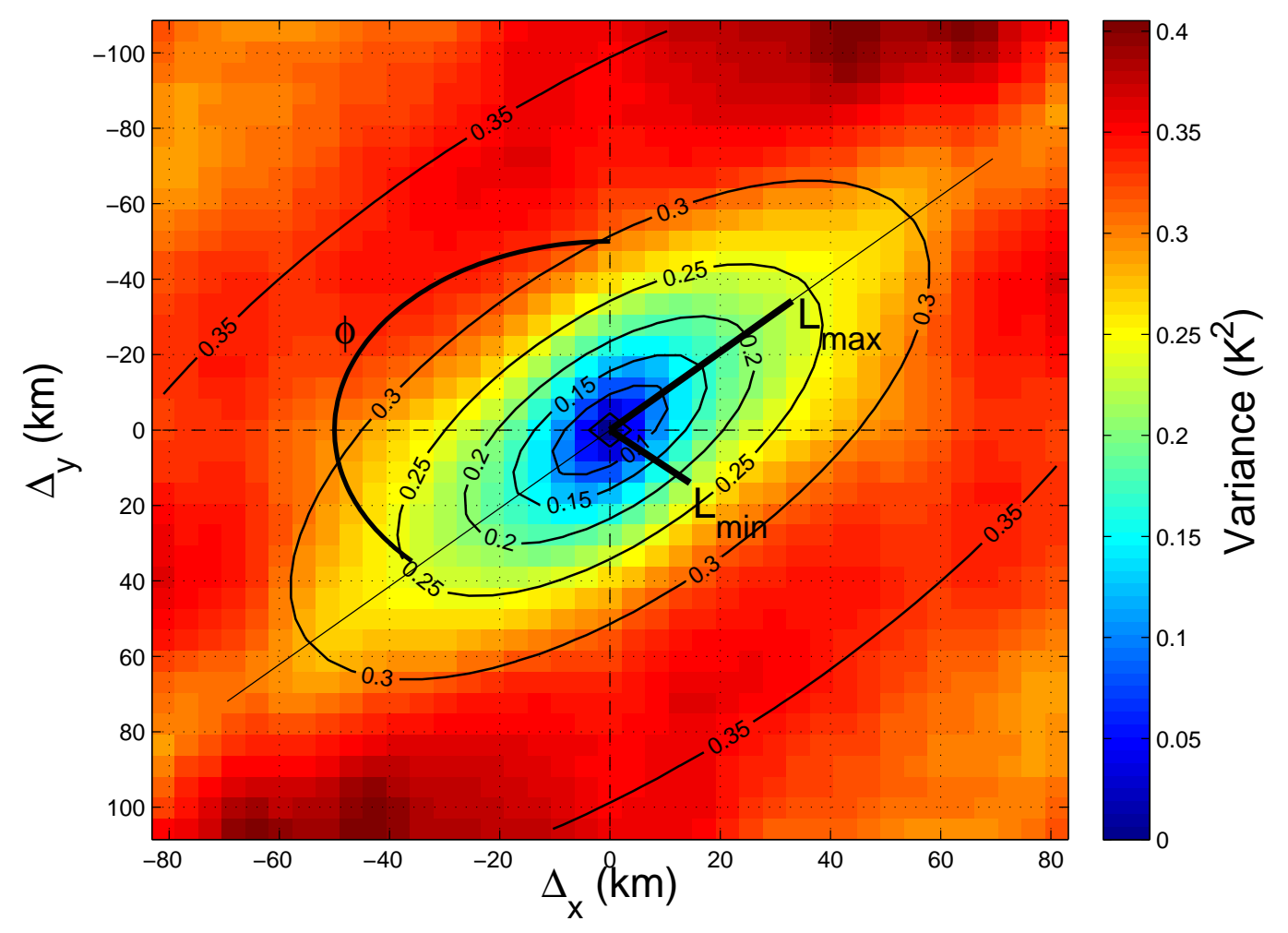

Fig. 2. 2008 cumulated empirical semivariogram (colors) of the SST anomaly and corresponding fitted anisotropic exponential model (contours) given in Eq. (5) in a $1^{\circ} \times 1^{\circ}$ region around $42{ }^{\circ} \mathrm{S}$ and $55^{\circ} \mathrm{W}$ in the Malvinas current off Argentina. The exponential anisotropic variability is represented as an ellipsoid with a minor range $L_{\min }$ and a major range $L_{\max }$ in the direction $\phi$.

within the considered area and retrieve the combinations of pairs in all the zonal and meridional directions such as described in Eq. (4). In a second step, the daily semivariograms are cumulated into a mean semivariogram over the year 2008. The mean is computed as a weighted version of the daily semivariograms where we account for the actual number of pairs of points separated by $\Delta_{x}$ and $\Delta_{y}$. As stated in Section 2, we fit to each empirical model a parametric exponential and anisotropic model given by Eq. (5) using a Gauss-Newton optimization and the weighted least squares criterion.

An example of empirical semivariogram is reported in Fig. 2 for a $1^{\circ} \times 1^{\circ}$ in the Malvinas current, off the Argentinian coasts. The empirical semivariogram is clearly anisotropic. This region involves a strong current which results in a low spatial SST variability along this direction. The corresponding fitted exponential semivariogram is illustrated as the contour plot. It might be noted that due to a larger number of pairs of points, the estimation of the empirical semivariogram is typically better for small spatial distance. The estimated model parameters in this particular region are given in Table 1.

We map in Fig. 3 the fitted model parameters for the Malvinas current. The 
Table 1

Estimated parameters of the anisotropic exponential semivariogram model of the SST anomaly in the $1^{\circ} \times 1^{\circ}$ region of the Malvinas current considered in Fig. 2, around $42{ }^{\circ} \mathrm{S}$ and $55^{\circ} \mathrm{W}$.

\begin{tabular}{cc}
\hline Parameter & Value \\
\hline Nugget $\sigma_{0}^{2}$ & $0.01 \mathrm{~K}^{2}$ \\
Sill $\sigma^{2}$ & $0.36 \mathrm{~K}^{2}$ \\
Minor range $L_{\min }$ & $20 \mathrm{~km}$ \\
Major range $L_{\max }$ & $47 \mathrm{~km}$ \\
Anisotropic angle $\phi$ & $152^{\circ}$ \\
\hline
\end{tabular}

estimated anisotropic spatial variability is represented by ellipses (the same representation was adopted in Morrow et al. (1994)). Each ellipse is characterized by its minor range $L_{\min }$, major range $L_{\max }$ and the angle of its anisotropy direction $\phi$. In addition, we report the stationary variability $\sigma^{2}$ as a color field. The visualization of the nugget effect will be reported only at the global scale in Fig. 4(c). The detailed analysis of the reported results for the Malvinas current clearly stress the spatial non-stationarity of the SST variability, both in terms of spatial correlation lengths and in terms of anisotropy factor. Especially, SST variability is greater near the Mar del Plata mouth and the estimated anisotropic angles $\phi$ are highly correlated to the mean annual currents velocities derived from the mean dynamic topography represented by arrows.

In the global Ocean, we plot the spatial distribution of the parameters in Fig. 4. From these maps, we retrieve relevant patterns regarding the spatial variability of SST and the quality of the METOP-AVHRR data in the global Ocean. First, in Fig. 4(a), the estimated stationary variance $\sigma^{2}$ of the SST anomaly highlights regions with strong surface currents depicting strong SST variabilities (from 1 to 1.5) such as the Malvinas, Kuroshio, Gulf Stream or Agulhas currents. In addition, we identify important upwelling systems (for instance, the Peruvian or Benguela systems) with strong winds yielding to important mixing of the upper ocean layer. By contrast, the mean variance in the rest of the global Ocean is about 0.1. Regarding the analysis of the anisotropy factor $0<=L_{\min } / L_{\max }<=1$ given in Fig. $4(\mathrm{~b})$, the reported results also clearly discriminates different ocean regions. The lower anisotropic factor, the greater the spatial anisotropy of the local SST variability. Conversely, anisotropic factors closer to 1 refers to almost isotropic situations. Highly dynamic regions appear as specific regions discriminated by their anisotropic spatial SST variabilities. As illustrated for the Malvinas region, the preferred direction relates to the mean surface currents. The Inter Tropical Convergence Zone (ITCZ) also depict intermediate mean anisotropy factors (between 0.4 and 0.6). By contrast, in other areas such as in the Northern Atlantic Ocean, 


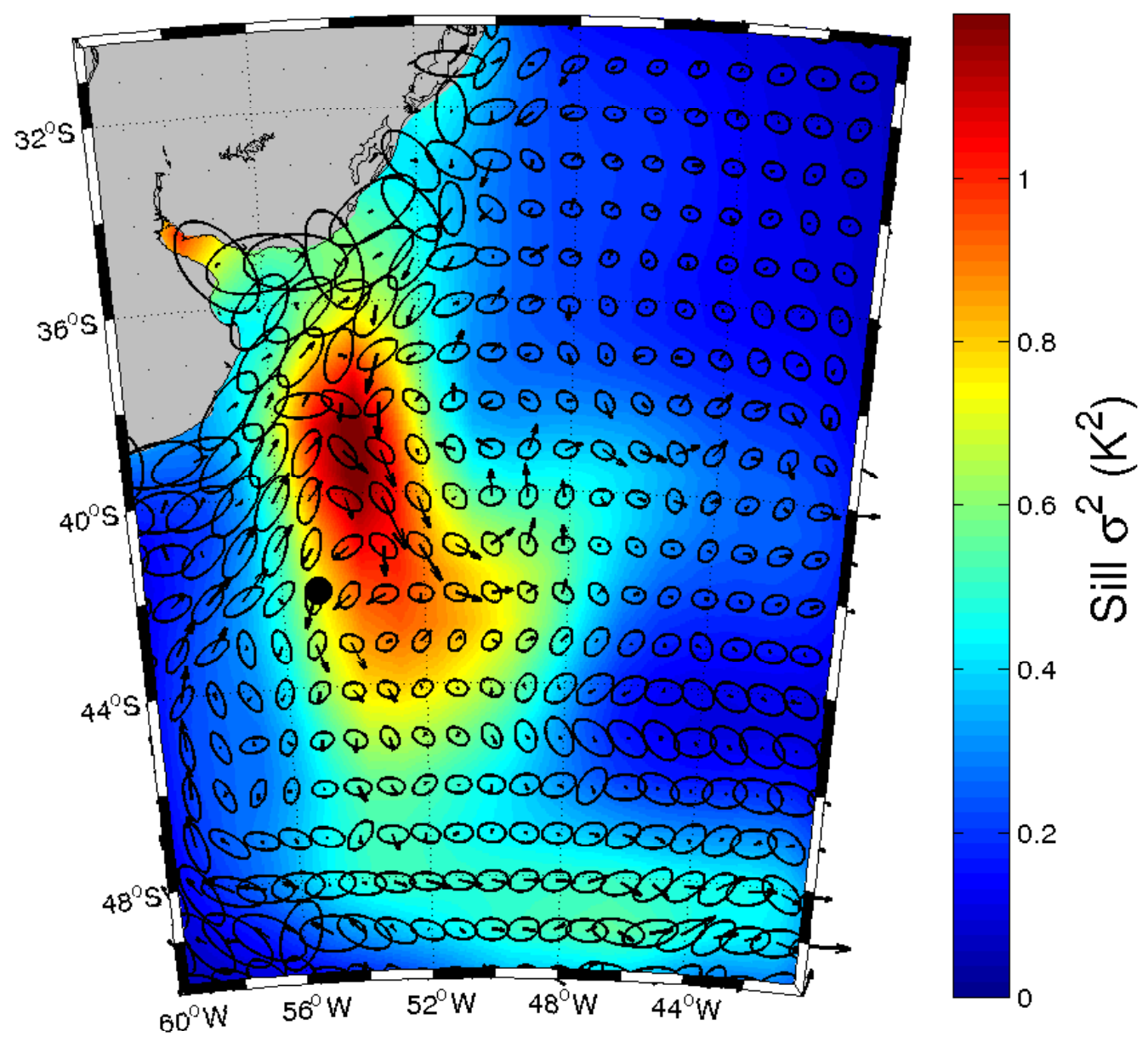

Fig. 3. Ellipse-based representation of the parametric semivariogram model given in Eq. (5) of the local $1^{\circ} \times 1^{\circ}$ variability of the SST anomaly in the Malvinas current. The minor and major of the ellipse depicts both the spatial correlation lengths $L_{\min }$ and $L_{\max }$. We report the sill $\sigma^{2}$ as a color field and the quivers refer to the mean geostrophic currents issues from the mean dynamic topography. The black dot corresponds to the locations of the anisotropic spatial semivariogram given in Fig. 2 .

we observe high levels of isotropy (more than 0.8), which indicate that a preferred direction of variation of the SST does not exist. In Fig. 4(c), we report the estimated map of the spatial nugget $\sigma_{0}^{2}$ parameters. The nugget parameter corresponds to the variance of the errors of measurement of the METOP-AVHRR sensor. Previous studies evaluated these variance levels from a comparison between the METOP-AVHRR SSTs and in situ drifting-buoy measurements (cf. Le Borgne et al. (2007)). Unfortunately, the number of buoys is very limited and estimates over a whole ocean cannot be determined. Here, the proposed approach only relies on remote sensing data, such that we can derive a synoptic view, at the ocean-scale, of the spatial distribution of this measurement uncertainties. The main sources of contamination of the METOP-AVHRR data documented in the literature are the Saharan dust 
(cf. Foltz and McPhaden (2008)), the water vapor in tropical regions such as the Indonesia waters and the Pacific Ocean (West of Colombia and Ecuador) as well as ice or cloud contamination at high latitudes. Such atmospheric processes or contaminations can produce regional biases between the METOP SST data and the OIV2 SST analysis. These biases have synoptic scales from 500 to $1500 \mathrm{~km}$ and can be considered as constant in $1^{\circ} \times 1^{\circ}$ boxes. Therefore, they do not affect the estimation of the semivariogram which represent the second order variability of the SST anomaly. However, a very local variability in the METOP-AVHRR SSTs measurements, known as the nugget effect, may be observed. The spatial extent of this nugget effect is clearly mapped in Fig. 4(c). Other regions such as the Gulf Stream and Agulhas currents also depict strong nugget effects highly correlated with the stationary variance $\sigma^{2}$. These strong nuggets may be due to sub-mesoscale physical structures at the surface of the ocean that cannot be resolved by the spatial resolution of the METOPAVHRR sensor. These results are also consistent with our previous temporal analysis of the METOP-AVHRR data (cf. Tandeo et al. (2011)), where the nugget effect was estimated in the Atlantic ocean. The anisotropic parameters are available at an interpolated $1^{\circ} \times 1^{\circ}$ spatial resolution as supplementary material and online at: ftp://ftp.ifremer.fr/ifremer/cersat/products/ gridded/sst-anisotropic-covariances/.

\section{Conclusion}

In this paper, we have presented a geostatistic method to characterize the non-stationarity of the spatial variability of the SST at mesoscale from 2008 METOP-AVHRR data. We chose an exponential model with an anisotropic parameterization to take into account the differences of the SST spatial variability along the directions. Five parameters were estimated in the global Ocean. They are available online as supplementary material. Among them, we find the stationary variance, the anisotropy parameters and the measurement error variance of the METOP-AVHRR SST data.

Among the key issues to be considered in future works, we plan to perform spatiotemporal interpolation of METOP-AVHRR data to provide realistic SST maps. Especially, we plan to use the statistical parameters of the temporal variability computed in Tandeo et al. (2011) and the spatial anisotropic parameters given in this paper. In this context, the anisotropic shape of the spatial variances might be an alternative to the classical isotropic assumptions in (i) the coastal and mouth regions, (ii) the front of SSTs and (iii) the strong surface currents.

Future work may also address the use of the full METOP-AVHRR archive to produce robust SST spatial variability parameters and to study their corre- 


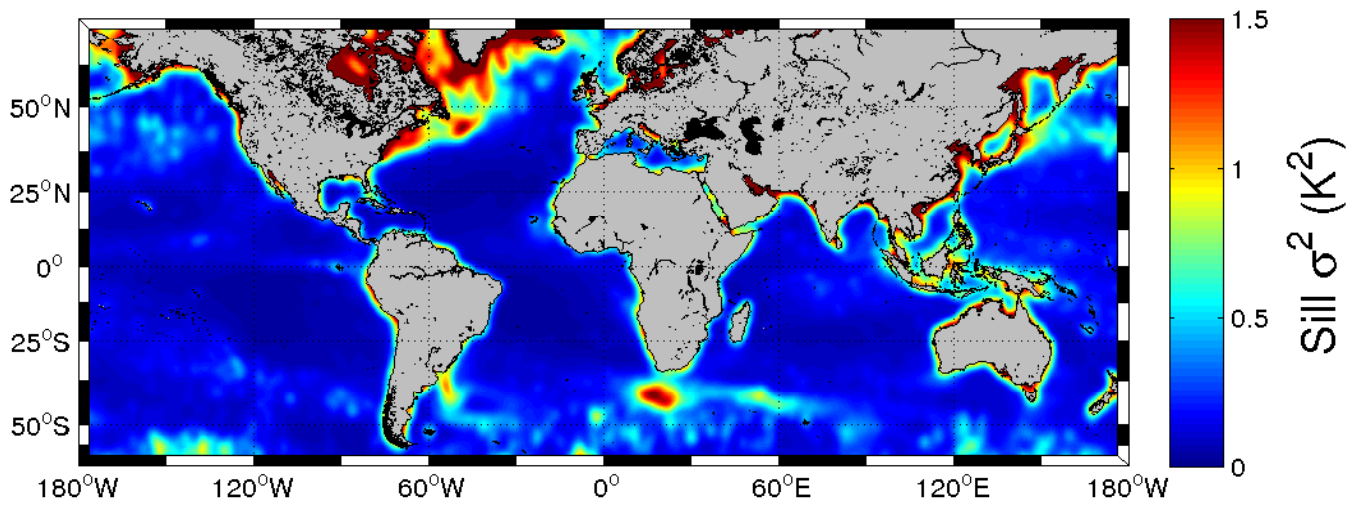

(a)

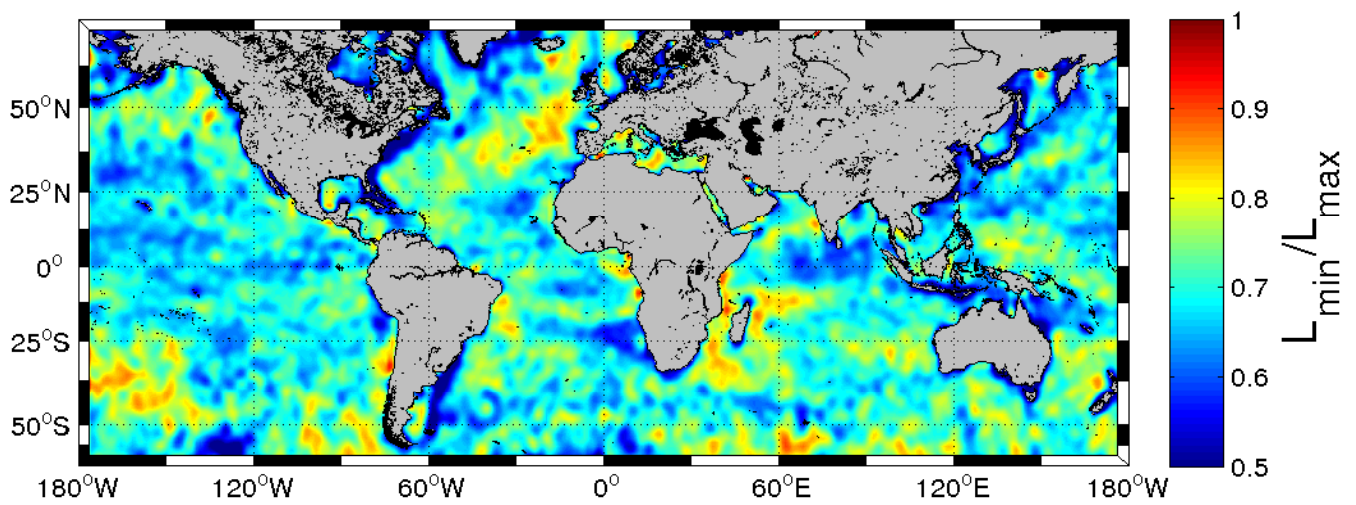

(b)

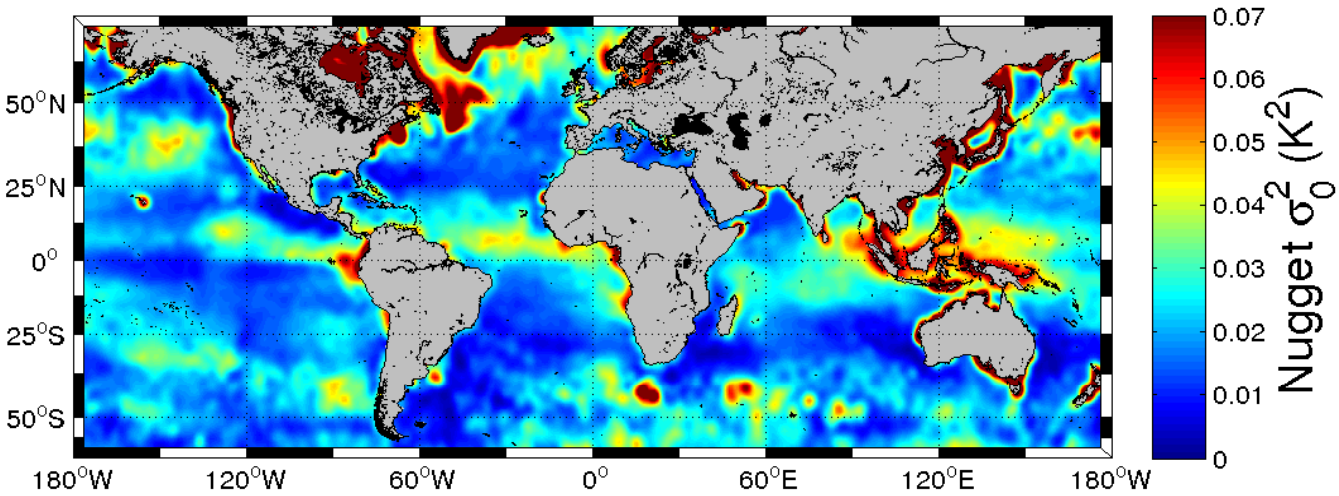

(c)

Fig. 4. Estimated parameters of the SST spatial variability in the global Ocean: (a) the sill parameter or the stationary variance $\sigma^{2},(\mathrm{~b})$ the anisotropic ratio $L_{\min } / L_{\max }$ and (c) the nugget effect or variance measurement error $\sigma_{0}^{2}$. These maps correspond to the fit of the parametric exponential and anisotropic semivariogram model given in Eq. (5) from the 2008 SST METOP-AVHRR data in $1^{\circ} \times 1^{\circ}$ areas over the global Ocean. 
sponding seasonal variations, especially over the major ocean current systems. The estimation of the parameters could be refined by introducing more flexible parametric models for the calculation of the theoretical semivariogram. For instance, one can use the Matérn (using a modified Bessel function, cf. Stein (1999), p. 31) or the powered-exponential functions (see discussion in Diggle and Ribeiro (2007), Section 3.4.2). The exponential and the Gaussian semivariograms are particular cases of these two functions. These models may be interpreted in term of stochastic operators, which might offer additional interpretations of the estimated parameters with respect to local advection/diffusion properties.

\section{Acknowledgment}

The data from the EUMETSAT Satellite Application Facility on Ocean and Sea Ice are accessible through the SAF's homepage: http://www.osi-saf . org/. We are grateful to Dr. Pierre Le Borgne for his expertise and valuable comments on this work.

\section{References}

Cressie, N. A. C. (1993). Statistics for Spatial Data, revised edition. New York, $U S A$.

Diggle, P. J., Ribeiro, P. J. (2007). Model-based geostatistics. New York, USA.

Doney, S. C., Glover, D. M., McCue, S. J., \& Fuentes, M. (2003). Mesoscale variability of SeaWiFS satellite ocean color: Global patterns and spatial scales. Journal of Geophysical Research, 108, 3024.

Donlon, C. J., Minnett, P. J., Gentemann, C., Nightingale, T. J., Barton, I. J., Ward, B., \& Murray, M. J. (2002). Toward improved validation of satellite sea surface skin temperature measurements for climate research. Journal of Climate, 15, 353-369.

Foltz, G. R., \& McPhaden, M. J. (2008). Impact of Saharan dust on tropical North Atlantic SST. Journal of Climate, 21, 5048-5060.

Gohin, F., \& Langlois, G. (1993). Using geostatistics to merge in situ measurements and remotely-sensed observations of sea surface temperature. International Journal of Remote Sensing, 14, 9-19.

Isern-Fontanet, J., Chapron, B., Lapeyre, G., \& Klein, P. (2006). Potential use of microwave sea surface temperatures for the estimation of ocean currents. Geophysical Research Letters, 33, L24608.

Klein, P., Isern-Fontanet, J., Lapeyre, G., Roullet, G., Danioux, E., Chapron, B., Le Gentil, S., \& Sasaki, H. (2009). Diagnosis of vertical velocities in the 
upper ocean from high resolution sea surface height. Geophysical Research Letters, 36, L12603.

Le Borgne, P., Legendre, G., \& Marsouin, A. (2007). Operational SST Retrieval from MetOp/AVHRR. Proceeding ot the 2007 EUMETSAT Conference, Amsterdam, the Netherlands.

Morrow, R., Coleman, R., Church, J., \& Chelton, D. (1994). Surface eddy momentum flux and velocity variances in the Southern Ocean from Geosat altimetry. Journal of Physical Oceanography, 24, 2050-2071.

O\&SI SAF Project Team. (2013). Low earth orbiter sea surface temperature product user manual. Technical report available at http: //www. osi-saf. org.

Park, K. A., \& Chung, J. Y. (1999). Spatial and temporal scale variations of sea surface temperature in the East Sea using NOAA/AVHRR data. Journal of Oceanography, 55, 271-288.

Reynolds, R. W., Smith, T. M., Liu, C., Chelton, D. B., Casey, K. S., \& Schlax, M. G. (2007). Daily High-Resolution-Blended Analyses for Sea Surface Temperature. Journal of Climate, 20, 5473-5496.

Stein, M. L. (1999). Interpolation of spatial data: some theory for kriging. New York, USA.

Tandeo, P., Ailliot, P., \& Autret, E. (2011). Linear Gaussian state-space model with irregular sampling: application to sea surface temperature. Stochastic Environmental Research and Risk Assessment, 25, 793-804.

Tandeo, P., Chapron, B., Ba, S., Autret, E., \& Fablet, R. (2013). Segmentation of mesoscale ocean surface dynamics using satellite SST and SSH observations. Geoscience and Remote Sensing, IEEE Transactions on, to appear.

Wainer, I., Clauzet, G., Servain, J., \& Soares, J. (2003). Time scales of upper ocean temperature variability inferred from the PIRATA data (1997-2000). Geophysical Research Letters, 30, 8004. 